\title{
The curing kinetic analysis of epoxy based on FT-IR
}

\author{
Ying $\mathrm{Fu}^{\mathrm{a}}$, Deshuai Sun ${ }^{\mathrm{b}}$, Xin Liuc, ${ }^{\mathrm{c}}$, Xingjie An ${ }^{\mathrm{d}}$, Xiaodong Zhang ${ }^{\mathrm{e},}$ \\ College of Chemical Science and Engineering, Qingdao University, Qingdao 266071, China

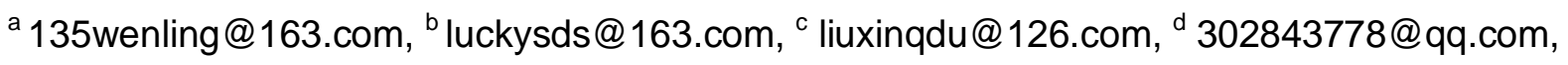 \\ e zhangxdqd@hotmail.com
}

Keywords: aromatic amine curing agents; aliphatic amine curing agents; curing process; first-order reaction

\begin{abstract}
The curing processes of epoxy resin cured with curing agents containing aromatic ring were analyzed quantitatively with FT-IR. The internal standard and baseline correction were used to decrease the analysis error. Due to the existence of aromatic ring in curing agent, the effects of curing agent dosage on absorbance at $1607 \mathrm{~cm}^{-1}$ were tested to calibrate the standard working curves. The built standard equation was used to analyze the curing kinetic of epoxy resin. The results showed that the curing percentage increased with the incremental of amount of curing agents. All curing reactions were all of first-order reaction. The curing reaction rates of curing agent T-31 were faster than that of curing agent TEPA and polyamide 650 .
\end{abstract}

\section{Introduction}

Epoxy resin has excellent physical properties, insulation properties and bonding properties, which is widely used in various fields. The properties of epoxy resin coatings are dependent on the curing conditions. FT-IR technique is used generally to investigate the curing processes of epoxy resin ${ }^{[1-4]}$, which can identify qualitatively the reaction group in curing reaction of epoxy resin. Some documents reported the quantitative curing processes of epoxy resin cured by aliphatic amine curing agents ${ }^{[2-4]}$ with FT-IR. However, no papers researched the epoxy curing kinetic with aromatic amine curing agents using FT-IR, because there were some aromatic ring in epoxy resin. In this paper, the curing processes of epoxy resin E-51 with aliphatic amine curing agents and aromatic amine were analyzed quantitatively using FT-IR on room temperature. In order to reduce the error of aromatic ring, internal standard and baseline correction were applied to build the standard working curves.

\section{Experimental}

Materials and Instruments. The chemicals included epoxy resin E-51 (epoxide number is 0.51), Curing agent polyamide 650, Curing agent T-31, and Curing agent tetraethylenepentamine (TEPA), which were industrial reagents. The structures of three curing agents were shown in Fig.1 and their physicochemical properties were shown in Table 1. Solvent 1,4-dioxane was an analytical reagent. Infrared spectroscopy (Nicolet FT-IR 300) was from Thermo Fisher Scientific.

Curing process of epoxy resin. $4.00 \mathrm{~g}$ E-51 and $16.00 \mathrm{~g}$ 1,4-dioxane were mixed and stirred at room temperature until a transparent solution was formed. The curing agent was then added and stirred for another 2 hours. After removing the air bubbles, the mixture solution containing $0.38 \mathrm{~g}$ E-51 was poured into a level culture dish with diameter of $6 \mathrm{~cm}$, which bottom was covered with a clean PE film (thickness $0.1 \mathrm{~mm}$ ). After a determined curing time at room temperature, the coating 
sample with PE film was prepared (the quantity of E-51 was calculated to be $0.0134 \mathrm{~g} / \mathrm{cm}^{2}$ ) and measured by IR. Each spectrum of the samples was collected in the $400 \mathrm{~cm}^{-1}$ to $2000 \mathrm{~cm}^{-1}$ region with $2 \mathrm{~cm}^{-1}$ resolution, 32 scans. All the results presented in the following sections were taken from an average of at least three experiments.

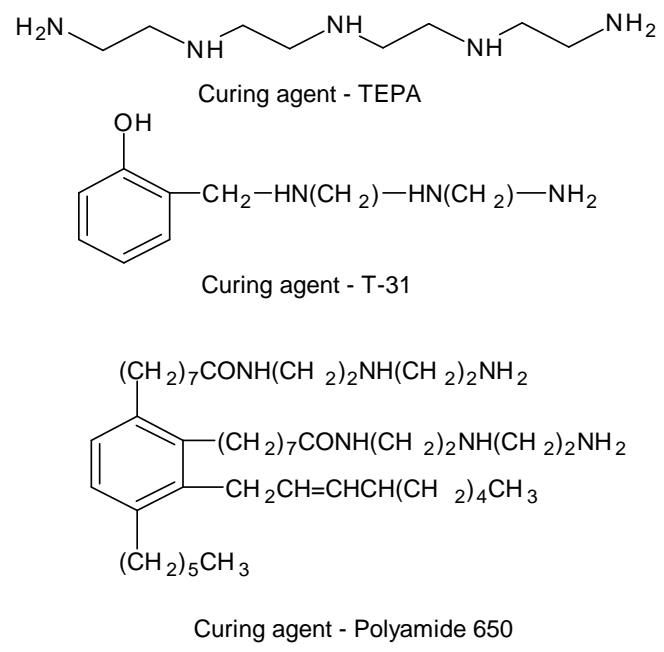

Table 1 Physicochemical properties of three curing agents

\begin{tabular}{lcc}
\hline $\begin{array}{l}\text { Curing } \\
\text { Agent }\end{array}$ & $\begin{array}{l}\text { Amine } \\
\text { value } \\
(\mathrm{mgKOH} / \mathrm{g})\end{array}$ & $\begin{array}{c}\text { Theoretical values } \\
\text { of curing E-51 } \\
(\mathrm{g} / 100 \mathrm{~g})\end{array}$ \\
\hline TEPA & 1484 & 13.77 \\
T-31 & 480 & 28.56 \\
Polyamide 650 & 220 & 102.00 \\
\hline
\end{tabular}

Fig.1 Structures of three curing agents

Test method. PE film was adhered on the bottom of the coating samples. The absorbance of PE film could affect the accuracy. Therefore, the FT-IR spectrum of PE film was measured and the values were subtracted as the baseline.

In the IR spectrum of epoxy resin, the peak at $914 \mathrm{~cm}^{-1}$ was characteristic absorption of epoxy groups and the strength decreased as the curing reaction proceeded. The characteristic absorption peak of benzene ring located at $1607 \mathrm{~cm}^{-1}$ and kept stable in curing process if the curing agent had no aromatic ring. In order to quantitatively analyze the curing extent of epoxy resin by IR, the internal standard method was taken in this research ${ }^{[5]}$. Internal standard was the characteristic absorption peak of benzene ring in at $1607 \mathrm{~cm}^{-1}$. Thus, the ratio of absorbance values at $914 \mathrm{~cm}^{-1}$ (marked as $\mathrm{A}_{914}$ ) and at $1607 \mathrm{~cm}^{-1}$ (marked as $\mathrm{A}_{1607}$ ) could be used to represent the conversion extent of epoxy groups. The curing degree of epoxy resin $(\alpha)$ could be calculated as Eq.(1) ${ }^{[2-4,6]}$

$\alpha=1-\left(\mathrm{A}_{914} / \mathrm{A}_{1607}\right)_{\mathrm{t}} /\left(\mathrm{A}_{914} / \mathrm{A}_{1607}\right)_{0}$

The curing degree of the epoxy resin could also be expressed as the changes of concentration of epoxy group. Therefore, $\alpha$ could also be calculated by the Eq. $(2)^{[2-3,6]}$ : $\alpha=1-\mathrm{C}_{\mathrm{t}} / \mathrm{C}_{0}$

Wherein $\left(\mathrm{A}_{914} / \mathrm{A}_{1607}\right)_{0}$ was the absorption ratio at the beginning of curing and $\left(\mathrm{A}_{914} / \mathrm{A}_{1607}\right)_{\mathrm{t}}$ was the absorption ratio at curing time $t$. The value $\mathrm{C}$ is the concentration of epoxy resin. The subscripts 0 and $t$ denote curing reaction time at zero and $t$, respectively.

\section{Results and discussion}

Standard working curves of the dosage of curing agents. As shown in Fig.1, there was aromatic ring in the molecular of T-31 or polyamide 650 . The absorption at $1607 \mathrm{~cm}^{-1}$ of the curing sample could increase with the incremental amount of curing agent. The strength at $914 \mathrm{~cm}^{-1} \mathrm{kept}$ stable due to constant amount of epoxy at the first time. Therefore, the standard working curves of 
needed to be calibrated. By changing the dosages of curing agents, the standard working curves that the dosages of curing agent dependence of absorbance strengths at peak $1607 \mathrm{~cm}^{-1}$ were shown in Fig.2. Because the correlation coefficient was higher than 0.99 , good linear relation could be build between the absorption and the amount of curing agent. According to this result, when curing agent T-31 or polyamide 650 was used in curing reaction, the Eq. (1) should be modified to Eq. (3).

$$
\mathrm{A}_{914} / \mathrm{A}_{1607}=\mathrm{A}_{914} /\left(\mathrm{A}_{1607-\mathrm{s}}-\mathrm{A}_{1607-\mathrm{b}}\right)
$$

Wherein $\mathrm{A}_{1607-\mathrm{S}}$ was the corrected absorbance from spectra of coating samples. $\mathrm{A}_{1607-\mathrm{b}}$ could be calculated according the equation from Fig.2.

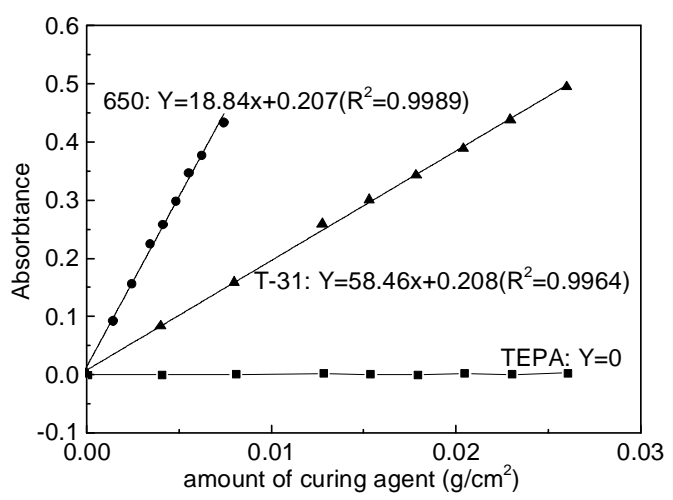

Fig.2 Dosages dependence of $A_{1607-b}$ of curing agents

The curing kinetics of epoxy resin. According to the standard curve, the curing kinetic could be calculated in different amount of curing agent. Fig.3 showed the calculated curing percentages of epoxy resin E-51. It could be found that $\alpha$ values increased with the prolongation of curing time and the incremental dosage of curing agents. The maximal curing percentages of epoxy E-51 were $98.32 \%$ for TEPA, $98.49 \%$ for T-31 and $96.98 \%$ for polyamide 650 in theoretical dosages, respectively. With increasing the amount of curing agents, the curing percentage increased. When the dosage was up to $180 \%$ of theoretical value for each of three curing agents, all three final $\alpha$ values were about $99.99 \%$.

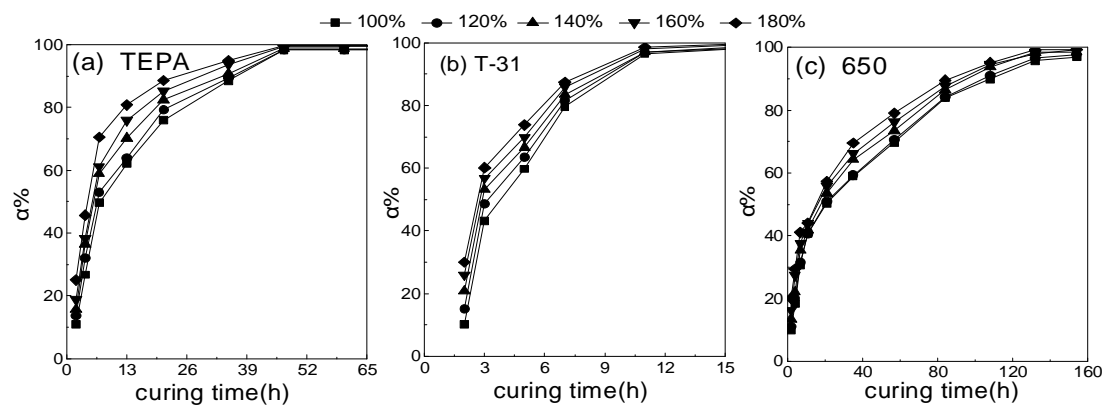

Fig.3 Curing percentages of epoxy cured by three curing agents

Fig.4 showed $\ln (1-\alpha)$-t fitting curves of the E-51 cured by three different curing agents. It could be seen there were good linear relations between $\ln (1-\alpha)$ and curing time $\left(R^{2} \geqslant 0.98\right)$, suggesting that the curing reactions are all of first-order reaction ${ }^{[6]}$. The absolute values of slope of $\ln (1-\alpha)$-t linear equation were the reaction rate constants of the curing reactions of E-51, which were shown in Table 2. From Table 2, it could be seen that all the rate constants increased with increasing the amount of curing agents, which indicated the curing reactions could be finished in shorter time. One also found the reaction rate of T-31 was significantly higher than that of TEPA and polyamide 650 . This could be explained the different structure of curing agent. 

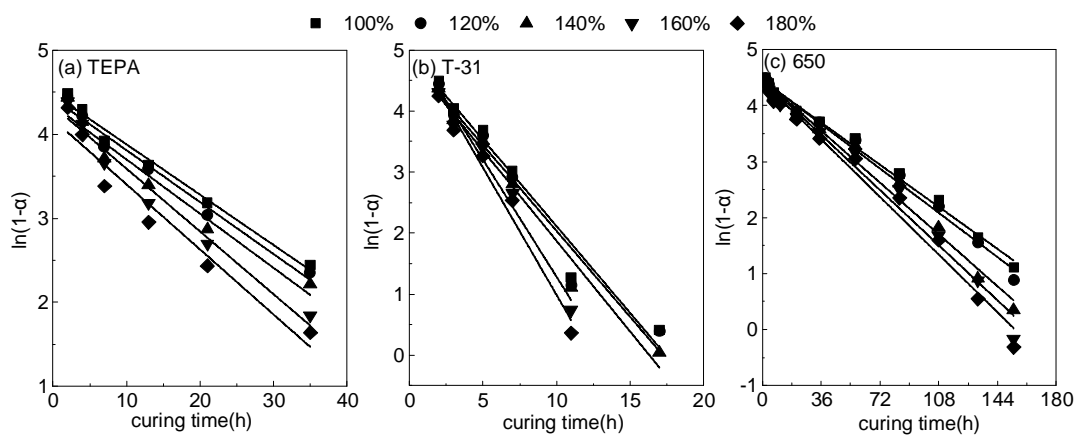

Fig.4 $\ln (1-\alpha)$-t fitting curves of the curing samples

Table 2 Reaction rate constant of epoxy/curing agents systems

\begin{tabular}{cccc}
\hline Dosage of curing agent & \multicolumn{3}{c}{$\mathrm{K}\left(\mathrm{h}^{-1}\right)$} \\
\cline { 2 - 4 } & $\mathrm{TEPA}$ & $\mathrm{T}-31$ & Polyamide 650 \\
\hline $100 \%$ & $0.05999\left(\mathrm{R}^{2}=0.988\right)$ & $0.28133\left(\mathrm{R}^{2}=0.981\right)$ & $0.02092\left(\mathrm{R}^{2}=0.991\right)$ \\
$120 \%$ & $0.06187\left(\mathrm{R}^{2}=0.981\right)$ & $0.28394\left(\mathrm{R}^{2}=0.983\right)$ & $0.02201\left(\mathrm{R}^{2}=0.989\right)$ \\
$140 \%$ & $0.06459\left(\mathrm{R}^{2}=0.980\right)$ & $0.29642\left(\mathrm{R}^{2}=0.982\right)$ & $0.02572\left(\mathrm{R}^{2}=0.985\right)$ \\
$160 \%$ & $0.07477\left(\mathrm{R}^{2}=0.982\right)$ & $0.38384\left(\mathrm{R}^{2}=0.980\right)$ & $0.02740\left(\mathrm{R}^{2}=0.980\right)$ \\
$180 \%$ & $0.07754\left(\mathrm{R}^{2}=0.980\right)$ & $0.41634\left(\mathrm{R}^{2}=0.981\right)$ & $0.02865\left(\mathrm{R}^{2}=0.982\right)$ \\
\hline
\end{tabular}

\section{Conclusions}

When there were some aromatic ring in curing agent, the absorption of benzene could affect the analysis of curing process with FT-IR. In order to decrease the analysis error, several methods including the internal standard, baseline correction and dosage correction were tested. The standard equation was used to analyze the curing process of epoxy resin. The maximal curing percentages of epoxy E-51 were $98.32 \%$ for TEPA, $98.49 \%$ for T-31 and $96.98 \%$ for polyamide 650 in theoretical dosages, respectively. With increasing the amount of curing agents, the curing percentage increased. All curing reactions were all of first-order reaction. The reaction rate of T-31 was significantly higher than that of TEPA and polyamide 650 .

\section{Acknowledgements}

The authors wish to acknowledge the financial support from the National Natural Science Foundation of China (Contract No. 21206080) and the Science and Technology development plan of Qingdao (Contract No. 12-1-4-2-(4)-jch).

\section{References}

[1] W. Zhang, A. A. Dehghani-Sanij, R. S. Blackburn, IR study on hydrogen bonding in epoxy resin-silica nanocomposites, Prog. Nat. Sci. 18 (2008) 801-805.

[2] N. Eidelman, D. Raghavan, A. M. Forster, E. J. Amis, A. Karim, Combinatorial approach to characterizing epoxy curing, Macromol. Rapid Commun. 25 (2004) 259-263.

[3] M. A. Escola, C. A. Moina, A. C. Nino Gomez, G. O.Ybarra, The determiation of the degree of cure in epoxy paints by infrared spectroscopy, Polym. Test. 24 (2005) 572-575.

[4] I. B. Recalde, D. Recalde, R. Garcia-Lopera, C. M. Gomez, FTIR isothermal cure kinetics and morphology of dicyanate ester resin/polysulfone blends, Eur. Polym. J. 41 (2005) 2635-2643.

[5] E. G. Karayannidu, D. S. Achilas, I. D. Sideridou, Cure Kinetics of epoxy-amine resin used in the restoration of works of art from glass or ceramic, Eur. Polym. J. 42 (2006) 3311-3323.

[6] W. G. Kim, J. Y. Lee, K. Y. Park, Curing reaction of $o$-cresol novolac epoxy resin according to 
hardener change, J. Polym. Sci. Pol. Chem. 31 (1993) 633-639. 